International Journal of Applied Mathematics

Volume 30 No. $5 \quad 2017,351-373$

ISSN: 1311-1728 (printed version); ISSN: 1314-8060 (on-line version)

doi: http://dx.doi.org/10.12732/ijam.v30i5.1

\title{
A POSTERIORI ERROR ESTIMATION FOR A DUAL MIXED FINITE ELEMENT METHOD FOR QUASI-NEWTONIAN FLOWS WHOSE VISCOSITY OBEYS A POWER LAW OR CARREAU LAW
}

\author{
Mohamed Farhloul ${ }^{1}$, Abdelmalek Zine ${ }^{2}$ \\ ${ }^{1}$ Département de Mathématiques et de Statistique \\ Université de Moncton \\ Moncton, N.B., E1A 3E9, CANADA \\ ${ }^{2}$ Département de Mathématiques et Informatique \\ Université de Lyon \\ Institut Camille Jordan, CNRS-UMR5208 \\ Ecole Centrale de Lyon, 36 av. Guy de Collongue \\ 69134 Ecully Cedex, FRANCE
}

\begin{abstract}
A dual mixed finite element method, for quasi-Newtonian fluid flow obeying the power law or the Carreau law, is constructed and analyzed in Farhloul-Zine [13]. This mixed formulation possesses good local (i.e., at element level) conservation properties (conservation of the momentum and the mass) as in the finite volume methods. In Farhloul-Zine [12], we developed an $a$ posteriori error analysis for a non-Newtonian fluid flow problems. The analysis is based on the fact that the equation describing the extra-stress tensor in terms of the rate of strain tensor is invertible and may give the rate of strain tensor as a function of the stress tensor. To free ourselves from this constraint of inversion of laws, and as a generalization of the obtained results in [12], we propose in this work an a posteriori error analysis to this mixed formulation.
\end{abstract}

AMS Subject Classification: 65N30, 65N15

Key Words: a posteriori error analysis, dual-mixed formulations, quasinewtonian, power law, Carreau law

Received: July 12, 2017

(C) 2017 Academic Publications

${ }^{\S}$ Correspondence author 


\section{Introduction}

Governed by the classical Stokes problem, the Newtonian fluid flows are a reasonable approximation of the more realistic non-Newtonian fluids (quasiNewtonian or Viscoelastic). In the case of quasi-Newtonian fluids, the viscosity is a function of strain rate tensor, temperature, time, etc. For a steady and creeping flow of an incompressible quasi-Newtonian fluid, the most used formulation, see Bird et al. [4], is based on the strain rate tensor. In that case, for $\Omega$ a bounded domain of $\mathbb{R}^{2}$ with a Lipschitz boundary $\Gamma$ and a given mass forces $\boldsymbol{f}$ defined on $\Omega$, the combination of the constitutive an conservation equations leads to the following nonlinear Stokes problem:

$$
\begin{cases}-\boldsymbol{d i v}(2 \mu(|\boldsymbol{d}(\boldsymbol{u})|) \boldsymbol{d}(\boldsymbol{u}))+\nabla p=\boldsymbol{f} & \text { in } \Omega, \\ \operatorname{div} \boldsymbol{u}=0 & \text { in } \Omega,\end{cases}
$$

where $\boldsymbol{u}$ and $p$, the unknowns of the problem, are the velocity and pressure, respectively. $\boldsymbol{d}(\boldsymbol{u})=\frac{1}{2}\left(\nabla \boldsymbol{u}+(\nabla \boldsymbol{u})^{t}\right)$ is the strain rate tensor, and $|\boldsymbol{d}(\boldsymbol{u})|^{2}=$ $\sum_{i, j=1}^{2} \boldsymbol{d}(\boldsymbol{u})_{i j}^{2}$.

For $\mu_{0}>0$ a reference viscosity and $r$ a fluid characteristic real parameter verifying $1<r<\infty$, the viscosity function $\mu(\cdot)$, depending on $|\boldsymbol{d}(\boldsymbol{u})|$, is usually given by one of the two following famous models:

$$
\begin{aligned}
& \mu(x)=\mu_{0} x^{r-2}, \forall x \in \mathbb{R}_{+}, \text {for the power law model, or } \\
& \mu(x)=\mu_{0}\left(1+x^{2}\right)^{(r-2) / 2}, \forall x \in \mathbb{R}_{+}, \text {for the Carreau model. }
\end{aligned}
$$

Finally, system (1) is supplemented by a set of boundary conditions.

The generalized Stokes problem (1) and its approximation by standard finite elements was first studied in Baranger and Najib [1]. Extensions and improvements of the error bounds have been obtained in Sandri [19] and Barrett and Liu $[2,3]$.

In these works, only the primal variables velocity and pressure are taken into account. But, for various reasons, one may need information on other (dual) variables such as velocity gradients $\nabla \boldsymbol{u}$, strain rate tensor $\boldsymbol{d}(\boldsymbol{u})$, and stress tensor $\boldsymbol{\sigma}=2 \mu(|\boldsymbol{d}(\boldsymbol{u})|) \boldsymbol{d}(\boldsymbol{u})$. For these reasons, it is necessary to build appropriate mixed formulations.

On the other hand, in connection with the use of the gradient tensor $\nabla \boldsymbol{u}$ which corresponds to the Ladyzhenskaya model [17]:

$$
\mu(|\nabla \boldsymbol{u}|)=\left(\mu_{0}+\mu_{1}|\nabla \boldsymbol{u}|\right)^{r-2}, \quad \mu_{0} \geq 0, \mu_{1}>0, r>1,
$$


a large amount of work is available in the literature. Among these works, there may be mentioned Manouzi and Farhloul [18], Farhloul and Zine [10], Gatica et al. [15, 16] and Ervin et al. [9]. The major drawback of formulations using the gradient lies in the fact that we can not deal with natural boundary conditions. To overcome this drawback related to the boundary conditions, we have introduced and analyzed a dual-mixed finite element method for quasiNewtonian fluid flow obeying to the power law, in Farhloul and Zine [11, 12]. A priori error estimates for the finite element approximation were proved in the first paper, while a posteriori error estimation was provided in the second work. In both papers, our analysis is based on the fact that the equation describing the extra-stress tensor in terms of the rate of strain tensor is invertible and give the rate of strain tensor as a function of the stress tensor. In a recent work Farhloul-Zine [13], we developed a mixed formulation to overcome this constraint of inversibility of the viscosity law. The main advantage of this formulation is that it makes it possible to consider differently viscosity functions obeying the power law or Carreau Law.

The aim of this work is to give an a posteriori error estimates for the mixed formulation developed in [13]. In the next Section 2 we recall the mixed formulation developed in [13] and then we give the a posteriori error estimates in Section 3. This will be done by extending our investigations by avoiding the assumption of expressing the rate of strain tensor as function of the stress tensor. We may be then able to deal with both problems associated with power law and Carreau model.

\section{Dual-Mixed Formulation}

In order to obtain a dual-mixed formulation of (1), first the problem (1) is formulated as follows:

$$
\begin{cases}-\boldsymbol{d i v}(\boldsymbol{\sigma}-p \boldsymbol{I})=\boldsymbol{f} & \text { in } \Omega, \\ \operatorname{div} \boldsymbol{u}=0 & \text { in } \Omega, \\ \boldsymbol{u}=0 & \text { on } \Gamma,\end{cases}
$$

and then, we introduce two new variables

$$
\begin{aligned}
\boldsymbol{t} & =\boldsymbol{d}(\boldsymbol{u}), \quad \text { the strain rate tensor, } \\
\mathcal{A}(\boldsymbol{t}) & =2 \mu(|\boldsymbol{t}|) \boldsymbol{t}=\boldsymbol{\sigma}, \quad \text { the stress tensor. }
\end{aligned}
$$

Let $1<r<\infty$ and $r^{\prime}$ being the conjugate number of $r$, i.e. $\frac{1}{r}+\frac{1}{r^{\prime}}=1$. Suppose $\boldsymbol{f} \in\left[L^{r^{\prime}}(\Omega)\right]^{2}$. Let $\boldsymbol{\omega}=\boldsymbol{\omega}(\boldsymbol{u})=\frac{1}{2}\left(\nabla \boldsymbol{u}-(\nabla \boldsymbol{u})^{t}\right)$ be the vorticity tensor. 
Then, for all $(\boldsymbol{\tau}, q) \in\left[L^{r^{\prime}}(\Omega)\right]^{2 \times 2} \times L_{0}^{r^{\prime}}(\Omega)$, such that $\boldsymbol{d i v}(\boldsymbol{\tau}-q \boldsymbol{I}) \in\left[L^{r^{\prime}}(\Omega)\right]^{2}$, and for all $\boldsymbol{u} \in\left[W^{1, r}(\Omega)\right]^{2}$ such that div $\boldsymbol{u}=0$, it is easy to see that

$$
(\boldsymbol{t}, \boldsymbol{\tau})=(\boldsymbol{d}(\boldsymbol{u}), \boldsymbol{\tau})=-(\operatorname{div}(\boldsymbol{\tau}-q \boldsymbol{I}), \boldsymbol{u})-(\boldsymbol{\omega}, \boldsymbol{\tau}),
$$

where, from now on $(\cdot, \cdot)$ denotes the duality pairing between $L^{p}(\Omega)$ and $L^{q}(\Omega), 1<p<\infty, q$ the conjugate of $p$, and

$$
L_{0}^{r^{\prime}}(\Omega)=\left\{q \in L^{r^{\prime}}(\Omega) ; \int_{\Omega} q d x=0\right\} .
$$

In order to derive the mixed formulation of (2), we define the following spaces:

$$
\begin{aligned}
\boldsymbol{T} & =\left[L^{r}(\Omega)\right]^{2 \times 2}, \\
\boldsymbol{\Sigma} & =\left\{(\boldsymbol{\tau}, q) \in\left[L^{r^{\prime}}(\Omega)\right]^{2 \times 2} \times L_{0}^{r^{\prime}}(\Omega) ; \boldsymbol{d i v}(\boldsymbol{\tau}-q \boldsymbol{I}) \in\left[L^{r^{\prime}}(\Omega)\right]^{2}\right\}, \\
\boldsymbol{M} & =\left\{(\boldsymbol{v}, \boldsymbol{\eta}) \in\left[L^{r}(\Omega)\right]^{2} \times\left[L^{r}(\Omega)\right]^{2 \times 2} ; \boldsymbol{\eta}+\boldsymbol{\eta}^{t}=0\right\},
\end{aligned}
$$

equipped with the following norms:

$$
\begin{aligned}
\|\boldsymbol{s}\|_{T} & =\|\boldsymbol{s}\|_{0, r, \Omega}=\left(\int_{\Omega}|\boldsymbol{s}|^{r}\right)^{\frac{1}{r}} \\
\|(\boldsymbol{\tau}, q)\|_{\Sigma} & =\left(\|\boldsymbol{\tau}\|_{0, r^{\prime}, \Omega}^{r^{\prime}}+\|q\|_{0, r^{\prime}, \Omega}^{r^{\prime}}+\|\boldsymbol{d i v}(\boldsymbol{\tau}-q \boldsymbol{I})\|_{0, r^{\prime}, \Omega}^{r^{\prime}}\right)^{\frac{1}{r^{\prime}}} \\
\|(\boldsymbol{v}, \boldsymbol{\eta})\|_{M} & =\left(\|\boldsymbol{v}\|_{0, r, \Omega}^{r}+\|\boldsymbol{\eta}\|_{0, r, \Omega}^{r}\right)^{\frac{1}{r}} .
\end{aligned}
$$

The dual-mixed formulation of problem (2) reads as follows: Find $\boldsymbol{t} \in \boldsymbol{T}$, $(\boldsymbol{\sigma}, p) \in \boldsymbol{\Sigma}$ and $(\boldsymbol{u}, \boldsymbol{\omega}) \in \boldsymbol{M}$ such that, $\forall \boldsymbol{s} \in \boldsymbol{T}, \forall(\boldsymbol{\tau}, q) \in \boldsymbol{\Sigma}$ and $\forall(\boldsymbol{v}, \boldsymbol{\eta}) \in \boldsymbol{M}$,

$$
\left\{\begin{array}{l}
(\mathcal{A}(\boldsymbol{t}), \boldsymbol{s})-(\boldsymbol{\sigma}, \boldsymbol{s})=0 \\
(\boldsymbol{t}, \boldsymbol{\tau})+(\boldsymbol{d i v}(\boldsymbol{\tau}-q \boldsymbol{I}), \boldsymbol{u})+(\boldsymbol{\tau}, \boldsymbol{\omega})=0 \\
(\boldsymbol{d i v}(\boldsymbol{\sigma}-p \boldsymbol{I}), \boldsymbol{v})+(\boldsymbol{\sigma}, \boldsymbol{\eta})+(\boldsymbol{f}, \boldsymbol{v})=0
\end{array}\right.
$$

Remark 1. From the last equation of (5),

$$
(\boldsymbol{d i v}(\boldsymbol{\sigma}-p \boldsymbol{I}), \boldsymbol{v})+(\boldsymbol{\sigma}, \boldsymbol{\eta})+(\boldsymbol{f}, \boldsymbol{v})=0, \quad \forall(\boldsymbol{v}, \boldsymbol{\eta}) \in \boldsymbol{M},
$$

one gets

$$
(\boldsymbol{\sigma}, \boldsymbol{\eta})=0, \forall \boldsymbol{\eta} \in\left[L^{r}(\Omega)\right]^{2 \times 2} \quad \text { such that } \boldsymbol{\eta}+\boldsymbol{\eta}^{t}=0 .
$$

This corresponds to the symmetry relaxation of the stress tensor $\sigma$ by a Lagrange multiplier. 
Remark 2. As stated above, the use of the rate of strain tensor enables to handle different types of boundary conditions, such as mixed boundary conditions. More precisely, assuming that we consider the following boundary conditions:

$$
\left\{\begin{array}{l}
\boldsymbol{u}=0 \quad \text { on } \Gamma_{D} \text { and } \\
(2 \mu(|\boldsymbol{d}(\boldsymbol{u})|) \boldsymbol{d}(\boldsymbol{u})-p \boldsymbol{I}) \quad \underline{\boldsymbol{n}}=0 \quad \text { on } \Gamma_{N},
\end{array}\right.
$$

where $\Gamma=\Gamma_{D} \cup \Gamma_{N}, \Gamma_{D} \neq \emptyset$ and $\underline{\boldsymbol{n}}$ is the unit outward normal vector field along the boundary of $\Omega$. Then, the only change to be made is to replace the space $\boldsymbol{\Sigma}$ by the following one:

$$
\begin{aligned}
\boldsymbol{\Sigma}=\{ & (\boldsymbol{\tau}, q) \in\left[L^{r^{\prime}}(\Omega)\right]^{2 \times 2} \times L_{0}^{r^{\prime}}(\Omega) ; \boldsymbol{d i v}(\boldsymbol{\tau}-q \boldsymbol{I}) \in\left[L^{r^{\prime}}(\Omega)\right]^{2} ; \\
& \left.(\boldsymbol{\tau}-q \boldsymbol{I}) \underline{\boldsymbol{n}}=0 \text { on } \Gamma_{N}\right\} .
\end{aligned}
$$

However, for the sake of clearness, we developed in [13] the analysis in the case of Dirichlet boundary conditions only.

Remark 3. Recall that the operator $\mathcal{A}$ is defined by

$$
\forall \boldsymbol{t} \in \boldsymbol{T}, \quad \mathcal{A}(\boldsymbol{t})=2 \mu(|\boldsymbol{t}|) \boldsymbol{t},
$$

$\mu$, the viscosity function, being given by either power or Carreau law.

The existence, uniqueness and stability of $(\boldsymbol{t}, \underset{\sim}{\boldsymbol{\sigma}}, \underset{\sim}{\boldsymbol{u}}) \in \boldsymbol{T} \times \boldsymbol{\Sigma} \times \boldsymbol{M}$, where $\underset{\sim}{\boldsymbol{\sigma}}=(\boldsymbol{\sigma}, p)$ and $\underset{\sim}{\boldsymbol{u}}=(\boldsymbol{u}, \boldsymbol{\omega})$, solution to the problem (5) are obtained in [13]. However, we recall here two inf-sup conditions in the continuous framework. These conditions are used for a posteriori estimates.

Lemma 4. There exists a positive constant $\beta$ such that

$$
\inf _{\underset{\sim}{\boldsymbol{v}} \in \boldsymbol{M}} \sup _{\underset{\sim}{\boldsymbol{\tau}} \in \boldsymbol{\Sigma}} \frac{(\boldsymbol{d i v}(\boldsymbol{\tau}-q \boldsymbol{I}), \boldsymbol{v})+(\boldsymbol{\tau}, \boldsymbol{\eta})}{\|\underset{\sim}{\boldsymbol{\tau}}\|_{\Sigma}\|\boldsymbol{\sim}\|_{M}} \geq \beta .
$$

Lemma 5. Let

$$
\boldsymbol{Z}^{*}=\left\{\boldsymbol{\tau}=(\boldsymbol{\tau}, q) \in \boldsymbol{\Sigma} ;(\boldsymbol{d i v}(\boldsymbol{\tau}-q \boldsymbol{I}), \boldsymbol{v})=0, \forall \boldsymbol{v} \in\left[L^{r}(\Omega)\right]^{2}\right\} .
$$

Then, there exists a positive constant $\beta^{*}$ such that

$$
\inf _{\underset{\sim}{\boldsymbol{\tau}} \in \boldsymbol{Z}^{*}} \sup _{\boldsymbol{s} \in \boldsymbol{T}} \frac{(\boldsymbol{s}, \boldsymbol{\tau})}{\|\boldsymbol{s}\|_{T}\|\boldsymbol{\tau}\|_{\Sigma}} \geq \beta^{*}
$$


Proof. The proof of this result is similar to the one of Lemma 3.6 in [13].

The following result giving the existence, uniqueness and stability is also established in [13].

Theorem 6. Problem (5) admits a unique solution $(\boldsymbol{t}, \underset{\sim}{\boldsymbol{\sigma}}, \underset{\sim}{\boldsymbol{u}}) \in \boldsymbol{T} \times \boldsymbol{\Sigma} \times \boldsymbol{M}$ satisfying the following stability condition,

$$
\|\boldsymbol{t}\|_{T}+\|\underset{\sim}{\boldsymbol{\sigma}}\|_{\Sigma}+\|\underset{\sim}{\boldsymbol{u}}\|_{M} \leq C(\boldsymbol{f})
$$

where $C(\boldsymbol{f})$ is a positive constant depending on $\boldsymbol{f}$.

Let us now recall the discrete problem. We assume that the boundary $\Gamma$ of the domain $\Omega$ is polygonal. We first give some finite element notations. Let $h>0$ and $\mathcal{T}_{h}$ a triangulation of $\Omega$ into triangles. We assume that the triangulation $\mathcal{T}_{h}$ is regular in the sense of Ciarlet [6]. Let $K \in \mathcal{T}_{h}$ be an element of the triangulation, we denote by $b_{K}$ the bubble function defined by

$$
b_{K}(x)=\lambda_{1}(x) \lambda_{2}(x) \lambda_{3}(x), \quad \forall x \in K,
$$

$\lambda_{i}, i=1, \cdots, 3$, being the barycentric co-ordinates with respect to the element $K$. For $k \in \mathbb{N}$, let $P_{k}(K)$ denote the space of polynomials of degree less than or equal to $k$ on $K$, and

$$
R(K)=\left[P_{1}(K)\right]^{2} \oplus \mathbb{R} \operatorname{curl} b_{K}
$$

where $\operatorname{curl} b_{K}=\left(\frac{\partial b_{K}}{\partial x_{2}},-\frac{\partial b_{K}}{\partial x_{1}}\right)$.

To write the discrete mixed formulation, we introduce the following finite dimensional spaces:

$$
\begin{gathered}
\boldsymbol{T}_{h}=\left\{\boldsymbol{s}_{h} \in \boldsymbol{T} ; \boldsymbol{s}_{\left.h\right|_{K}} \in R(K), \forall K \in \mathcal{T}_{h}\right\}, \\
\boldsymbol{\Sigma}_{h}=\left\{\boldsymbol{\tau}_{\sim}=\left(\boldsymbol{\tau}_{h}, q_{h}\right) \in \boldsymbol{\Sigma} ; \boldsymbol{\tau}_{\left.h\right|_{K}} \in[R(K)]^{2},\right. \\
\left.q_{\left.h\right|_{K}} \in P_{1}(K), \forall K \in \mathcal{T}_{h}\right\}, \\
\boldsymbol{M}_{h}=\left\{\underset{\sim}{\boldsymbol{v}_{\boldsymbol{h}}}=\left(\boldsymbol{v}_{h}, \boldsymbol{\eta}_{h}\right) \in \boldsymbol{M} ; \boldsymbol{v}_{\left.h\right|_{K}} \in\left[P_{0}(K)\right]^{2}, \boldsymbol{\eta}_{h}=\theta_{h} \boldsymbol{\chi},\right. \\
\left.\theta_{\left.h\right|_{K}} \in P_{1}(K), \forall K \in \mathcal{T}_{h}\right\},
\end{gathered}
$$


where

$$
\chi=\left[\begin{array}{rr}
0 & -1 \\
1 & 0
\end{array}\right]
$$

The discrete mixed formulation of problem (5) is given by the following: Find $\boldsymbol{t}_{h} \in \boldsymbol{T}_{h}, \underset{\sim}{\boldsymbol{\sigma}_{h}} \in \boldsymbol{\Sigma}_{h}$ and $\underset{\sim}{\boldsymbol{u}_{\boldsymbol{h}}} \in \boldsymbol{M}_{h}$ such that, $\forall \boldsymbol{s}_{h} \in \boldsymbol{T}_{h}, \underset{\sim}{\forall \boldsymbol{\tau}_{h}}=\left(\boldsymbol{\tau}_{h}, q_{h}\right) \in \boldsymbol{\Sigma}_{h}$ and $\underset{\sim}{\forall \boldsymbol{v}_{h}}=\left(\boldsymbol{v}_{h}, \boldsymbol{\eta}_{h}\right) \in \boldsymbol{M}_{h}$,

$$
\left\{\begin{array}{l}
\left(\mathcal{A}\left(\boldsymbol{t}_{h}\right), \boldsymbol{s}_{h}\right)-\left(\boldsymbol{\sigma}_{h}, \boldsymbol{s}_{h}\right)=0 \\
\left(\boldsymbol{t}_{h}, \boldsymbol{\tau}_{h}\right)+\left(\boldsymbol{d i v}\left(\boldsymbol{\tau}_{h}-q_{h} \boldsymbol{I}\right), \boldsymbol{u}_{h}\right)+\left(\boldsymbol{\tau}_{h}, \boldsymbol{\omega}_{h}\right)=0 \\
\left(\boldsymbol{d i v}\left(\boldsymbol{\sigma}_{h}-p_{h} \boldsymbol{I}\right), \boldsymbol{v}_{h}\right)+\left(\boldsymbol{\sigma}_{h}, \boldsymbol{\eta}_{h}\right)+\left(\boldsymbol{f}, \boldsymbol{v}_{h}\right)=0
\end{array}\right.
$$

The analysis of the above discrete problem (7), as well as the a priori estimates of discrete errors, are given in Farhloul-Zine [13]. We recall here some of theses results.

Lemma 7. Let

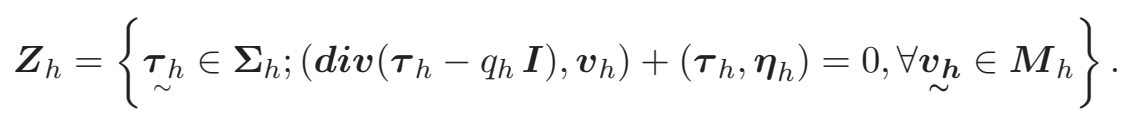

Then, there exists a positive constant $C$, independent of $h$, such that

$$
\forall \boldsymbol{\tau}_{\boldsymbol{\sim}} \in \boldsymbol{Z}_{h}, \quad\left\|q_{h}\right\|_{0, r^{\prime}, \Omega} \leq C\left\|\boldsymbol{\tau}_{h}\right\|_{0, r^{\prime}, \Omega}
$$

We have, as for the continuous problem, the existence, uniqueness and stability of the discrete solution. More precisely, the following theorem holds.

Theorem 8. Problem (7) admits a unique solution

$$
\left(\boldsymbol{t}_{h}, \boldsymbol{\sigma}_{\boldsymbol{\sim}}, \underset{\sim}{\boldsymbol{u}_{h}}\right) \in \boldsymbol{T}_{h} \times \boldsymbol{\Sigma}_{h} \times \boldsymbol{M}_{h}
$$

satisfying the following stability condition,

$$
\left\|\boldsymbol{t}_{h}\right\|_{T}+\left\|\underset{\sim}{\boldsymbol{\sigma}_{\boldsymbol{h}}}\right\|_{\Sigma}+\left\|\underset{\sim}{\boldsymbol{u}_{\boldsymbol{h}}}\right\|_{M} \leq C(\boldsymbol{f}),
$$

where $C(\boldsymbol{f})$ is a positive constant depending on $\boldsymbol{f}$ and independent of $h$. 


\section{A posteriori Error Estimates}

Let $(\boldsymbol{t}, \underset{\sim}{\boldsymbol{\sigma}}, \underset{\sim}{\boldsymbol{u}})=(\boldsymbol{t},(\boldsymbol{\sigma}, p),(\boldsymbol{u}, \boldsymbol{\omega})) \in \boldsymbol{T} \times \boldsymbol{\Sigma} \times \boldsymbol{M}$ and

$$
\left(\boldsymbol{t}_{h}, \boldsymbol{\sigma}_{\sim}, \underset{\sim}{\boldsymbol{u}_{h}}\right)=\left(\boldsymbol{t}_{h},\left(\boldsymbol{\sigma}_{h}, p_{h}\right),\left(\boldsymbol{u}_{h}, \boldsymbol{\omega}_{h}\right)\right) \in \boldsymbol{T}_{h} \times \boldsymbol{\Sigma}_{h} \times \boldsymbol{M}_{h}
$$

be the solutions of (5) and (7), respectively. On $\boldsymbol{T}, \boldsymbol{\Sigma}$ and $\boldsymbol{M}$, one define the residuals $\boldsymbol{R}_{1}, \boldsymbol{R}_{2}$ and $\boldsymbol{R}_{3}$ by, $\forall \boldsymbol{s} \in \boldsymbol{T}, \forall \underset{\sim}{\boldsymbol{\tau}}=(\boldsymbol{\tau}, q) \in \boldsymbol{\Sigma}$ and $\forall \underset{\sim}{\boldsymbol{v}}=(\boldsymbol{v}, \boldsymbol{\eta}) \in \boldsymbol{M}$,

$$
\begin{aligned}
& <\boldsymbol{R}_{1}, \boldsymbol{s}>=\left(\mathcal{A}\left(\boldsymbol{t}_{h}\right), \boldsymbol{s}\right)-\left(\boldsymbol{\sigma}_{h}, \boldsymbol{s}\right), \\
& <\boldsymbol{R}_{2}, \underset{\sim}{\boldsymbol{\tau}}>=\left(\boldsymbol{t}_{h}, \boldsymbol{\tau}\right)+\left(\boldsymbol{d i v}(\boldsymbol{\tau}-q \boldsymbol{I}), \boldsymbol{u}_{h}\right)+\left(\boldsymbol{\tau}, \boldsymbol{\omega}_{h}\right), \\
& <\boldsymbol{R}_{3}, \underset{\sim}{\boldsymbol{v}}>=\left(\boldsymbol{d i v}\left(\boldsymbol{\sigma}_{h}-p_{h} \boldsymbol{I}\right), \boldsymbol{v}\right)+\left(\boldsymbol{\sigma}_{h}, \boldsymbol{\eta}\right)+(\boldsymbol{f}, \boldsymbol{v}) .
\end{aligned}
$$

We denote by $\boldsymbol{R}_{1 *}, \boldsymbol{R}_{2 *}$ and $\boldsymbol{R}_{3 *}$ the dual norms of $\boldsymbol{R}_{1}, \boldsymbol{R}_{2}$ and $\boldsymbol{R}_{3}$, respectively.

$$
\begin{aligned}
& \boldsymbol{R}_{1 *}=\sup _{\boldsymbol{s} \in \boldsymbol{T}} \frac{\left|<\boldsymbol{R}_{1}, \boldsymbol{s}>\right|}{\|\boldsymbol{s}\|_{T}}, \\
& \boldsymbol{R}_{2 *}=\sup _{\underset{\sim}{\boldsymbol{\tau}} \in \boldsymbol{\Sigma}} \frac{\left|<\boldsymbol{R}_{2}, \underset{\sim}{\boldsymbol{\tau}}>\right|}{\|\boldsymbol{\tau}\|_{\Sigma}},
\end{aligned}
$$

and

$$
\boldsymbol{R}_{3 *}=\sup _{\underset{\sim}{\boldsymbol{v}} \in \boldsymbol{M}} \frac{\left|<\boldsymbol{R}_{3}, \underset{\sim}{\boldsymbol{v}}>\right|}{\|\underset{\sim}{\boldsymbol{v}}\|_{M}} .
$$

In the sequel, our goal is to obtain upper bounds of the errors $\left\|\boldsymbol{t}-\boldsymbol{t}_{h}\right\|_{T}$, $\left\|\underset{\sim}{\boldsymbol{\sigma}}-\boldsymbol{\sigma}_{\sim}\right\|_{\Sigma}$ and $\left\|\underset{\sim}{\boldsymbol{u}}-{\underset{\sim}{\boldsymbol{u}}}_{h}\right\|_{M}$ as functions of the above dual norms $\boldsymbol{R}_{1 *}, \boldsymbol{R}_{2 *}$ and $\boldsymbol{R}_{3 *}$ whose expressions involve only the data of the problem and the available computed quantities $\boldsymbol{t}_{h}, \boldsymbol{\sigma}_{\sim}$ and $\boldsymbol{u}_{\sim}$. And then, we give upper bounds to the estimators $\boldsymbol{R}_{1 *}, \boldsymbol{R}_{2 *}$ and $\boldsymbol{R}_{3 *}$.

As we will see later, these results depend on the parameter $r$. We have then to distinguish two cases:

$$
1<r<2 \text { and } r \geq 2 \text {. }
$$

First, let us give the following estimate of $\left(\mathcal{A}\left(\boldsymbol{t}_{h}\right)-\mathcal{A}(\boldsymbol{t}), \boldsymbol{t}_{h}-\boldsymbol{t}\right)$ in terms of $\boldsymbol{R}_{1 *}, \boldsymbol{R}_{2 *}$ and $\boldsymbol{R}_{3 *}$. 
Proposition 9. There exists a constant $C$ independent of $h$ such that

$$
\begin{aligned}
\left(\mathcal{A}\left(\boldsymbol{t}_{h}\right)-\mathcal{A}(\boldsymbol{t}), \boldsymbol{t}_{h}-\boldsymbol{t}\right) \leq C\left\{\left(\boldsymbol{R}_{1 *}+\boldsymbol{R}_{3 *}\right)\left\|\boldsymbol{t}_{h}-\boldsymbol{t}\right\|_{T}\right. \\
+\boldsymbol{R}_{2 *}\left\|\boldsymbol{f}-P_{h}^{0} \boldsymbol{f}\right\|_{0, r^{\prime}, \Omega}+\left(\boldsymbol{R}_{1 *}+\boldsymbol{R}_{3 *}\right) \boldsymbol{R}_{2 *} \\
\left.\quad+\boldsymbol{R}_{2 *} \sup _{\boldsymbol{s} \in \boldsymbol{T}} \frac{\left(\mathcal{A}(\boldsymbol{t})-\mathcal{A}\left(\boldsymbol{t}_{h}\right), \boldsymbol{s}\right)}{\|\boldsymbol{s}\|_{T}}\right\},
\end{aligned}
$$

where $P_{h}^{0} \boldsymbol{f}$ is the $L^{2}$-projection of $\boldsymbol{f}$ onto $\left[\prod_{K \in \mathcal{T}_{h}} P_{0}(K)\right]^{2}$.

Proof. From the first equation of (5), one gets, $\forall \boldsymbol{s} \in \boldsymbol{T}$,

$$
\begin{aligned}
\left(\mathcal{A}\left(\boldsymbol{t}_{h}\right)-\mathcal{A}(\boldsymbol{t}), s\right) & =\left(\mathcal{A}\left(\boldsymbol{t}_{h}\right), s\right)-(\boldsymbol{\sigma}, s) \\
& =\left(\mathcal{A}\left(\boldsymbol{t}_{h}\right), s\right)-\left(\boldsymbol{\sigma}_{h}, s\right)+\left(\boldsymbol{\sigma}_{h}, s\right)-(\boldsymbol{\sigma}, s) \\
& =\left\langle\boldsymbol{R}_{1}, s\right\rangle+\left(\boldsymbol{\sigma}_{h}-\boldsymbol{\sigma}, \boldsymbol{s}\right) .
\end{aligned}
$$

Thus,

$$
\left(\mathcal{A}\left(\boldsymbol{t}_{h}\right)-\mathcal{A}(\boldsymbol{t}), s\right)=\left\langle\boldsymbol{R}_{1}, s\right\rangle+\left(\boldsymbol{\sigma}_{h}-\boldsymbol{\sigma}, \boldsymbol{s}\right), \quad \forall s \in \boldsymbol{T} .
$$

From the second equation of (5), one gets, $\forall \underset{\sim}{\boldsymbol{\tau}} \in \boldsymbol{\Sigma}$,

$$
\left(\boldsymbol{t}_{h}-\boldsymbol{t}, \boldsymbol{\tau}\right)+\left(\operatorname{div}(\boldsymbol{\tau}-q \boldsymbol{I}), \boldsymbol{u}_{h}-\boldsymbol{u}\right)+\left(\boldsymbol{\tau}, \boldsymbol{\omega}_{h}-\boldsymbol{\omega}\right)=\left\langle\boldsymbol{R}_{2}, \boldsymbol{\tau}\right\rangle .
$$

Using the last equation of (5), we obtain, $\forall \underset{\sim}{\boldsymbol{v}} \in \boldsymbol{M}$,

$$
\left.\left(\boldsymbol{d i v}\left[\left(\boldsymbol{\sigma}_{h}-p_{h} \boldsymbol{I}\right)-(\boldsymbol{\sigma}-p \boldsymbol{I})\right], \boldsymbol{v}\right)+\left(\boldsymbol{\sigma}_{h}-\boldsymbol{\sigma}, \boldsymbol{\eta}\right)\right)=\left\langle\boldsymbol{R}_{3}, \underset{\sim}{\boldsymbol{v}}\right\rangle .
$$

Now, taking $\underset{\sim}{\boldsymbol{\tau}}=\boldsymbol{\sigma}_{\sim}-\underset{\sim}{\boldsymbol{\sigma}}$ in (14) and $\underset{\sim}{\boldsymbol{v}}=\boldsymbol{\sim}_{\sim}-\underset{\sim}{\boldsymbol{u}}$ in (15), we obtain

$$
\begin{aligned}
\left(\boldsymbol{t}_{h}-\boldsymbol{t}, \boldsymbol{\sigma}_{h}-\boldsymbol{\sigma}\right)+ & \left(\boldsymbol{d i v}\left[\left(\boldsymbol{\sigma}_{h}-p_{h} \boldsymbol{I}\right)-(\boldsymbol{\sigma}-p \boldsymbol{I})\right], \boldsymbol{u}_{h}-\boldsymbol{u}\right) \\
& +\left(\boldsymbol{\sigma}_{h}-\boldsymbol{\sigma}, \boldsymbol{\omega}_{h}-\boldsymbol{\omega}\right)=\left\langle\boldsymbol{R}_{2}, \boldsymbol{\sigma}_{h}-\underset{\sim}{\boldsymbol{\sigma}}\right\rangle
\end{aligned}
$$

and,

$$
\begin{gathered}
\left(\boldsymbol{d i v}\left[\left(\boldsymbol{\sigma}_{h}-p_{h} \boldsymbol{I}\right)-(\boldsymbol{\sigma}-p \boldsymbol{I})\right],\right. \\
\left., \boldsymbol{u}_{h}-\boldsymbol{u}\right)+\left(\boldsymbol{\sigma}_{h}-\boldsymbol{\sigma}, \boldsymbol{\omega}_{h}-\boldsymbol{\omega}\right) \\
=\left\langle\boldsymbol{R}_{3}, \boldsymbol{u}_{h}-\underset{\sim}{\boldsymbol{u}}\right\rangle .
\end{gathered}
$$

These two last equations imply

$$
\left(\boldsymbol{t}_{h}-\boldsymbol{t}, \boldsymbol{\sigma}_{h}-\boldsymbol{\sigma}\right)=\left\langle\boldsymbol{R}_{2}, \boldsymbol{\sigma}_{\sim}-\underset{\sim}{\boldsymbol{\sigma}}\right\rangle-\left\langle\boldsymbol{R}_{3}, \underset{\sim}{\boldsymbol{u}_{h}}-\underset{\sim}{\boldsymbol{u}}\right\rangle .
$$


Finally, substituting $\boldsymbol{s}$, by $\boldsymbol{s}=\boldsymbol{t}_{h}-\boldsymbol{t}$, in (13) and using the above last equation, we get

$$
\begin{aligned}
\left(\mathcal{A}\left(\boldsymbol{t}_{h}\right)-\mathcal{A}(\boldsymbol{t}), \boldsymbol{t}_{h}-\boldsymbol{t}\right)=\left\langle\boldsymbol{R}_{1}, \boldsymbol{t}_{h}-\boldsymbol{t}\right\rangle \\
+\left\langle\boldsymbol{R}_{2}, \boldsymbol{\sigma}_{\sim}-\underset{\sim}{\boldsymbol{\sigma}}\right\rangle-\left\langle\boldsymbol{R}_{3}, \underset{\sim}{\left.\boldsymbol{u}_{h}-\underset{\sim}{\boldsymbol{u}}\right\rangle .}\right.
\end{aligned}
$$

By the inf-sup condition (6) and (14), it follows

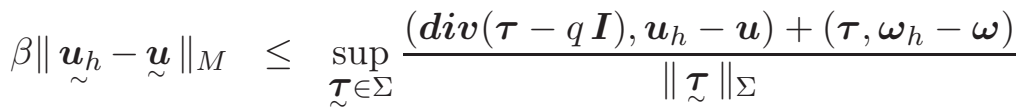

$$
\begin{aligned}
& \leq \sup _{\underset{\sim}{\boldsymbol{\tau}} \in \Sigma} \frac{\left\langle\boldsymbol{R}_{2}, \boldsymbol{\tau}>\right.}{\|\boldsymbol{\tau}\|_{\Sigma}}+\sup _{\underset{\sim}{\boldsymbol{\tau}} \in \Sigma} \frac{\left|\left(\boldsymbol{t}_{h}-\boldsymbol{t}, \boldsymbol{\tau}\right)\right|}{\|\boldsymbol{\tau}\|_{\Sigma}}
\end{aligned}
$$

Thus,

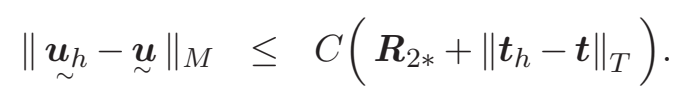

Now, using the third equation of (5) and the third equation of (7), one gets, $\forall \boldsymbol{v} \in\left[L^{r}(\Omega)\right]^{2}$,

$$
\begin{aligned}
\left(\boldsymbol { d i v } \left[\left(\boldsymbol{\sigma}_{h}-p_{h} \boldsymbol{I}\right)-\right.\right. & (\boldsymbol{\sigma}-p \boldsymbol{I})], \boldsymbol{v})+\left(P_{h}^{0} \boldsymbol{f}-\boldsymbol{f}, \boldsymbol{v}\right) \\
= & \left(\boldsymbol{d i v}\left(\boldsymbol{\sigma}_{h}-p_{h} \boldsymbol{I}\right)+P_{h}^{0} \boldsymbol{f}, \boldsymbol{v}\right)=0 .
\end{aligned}
$$

On the other hand, since $P_{h}^{0} \boldsymbol{f}-\boldsymbol{f} \in\left[L_{0}^{r^{\prime}}(\Omega)\right]^{2}$, there exists (see Galdi [14])

$$
\boldsymbol{\xi} \in\left\{\boldsymbol{\tau} \in\left[L^{r^{\prime}}(\Omega)\right]^{2 \times 2} ; \boldsymbol{d i v} \boldsymbol{\tau} \in\left[L^{r^{\prime}}(\Omega)\right]^{2}\right\}
$$

such that $\boldsymbol{d i v} \boldsymbol{\xi}=P_{h}^{0} \boldsymbol{f}-\boldsymbol{f}$ in $\Omega$ and $\|\boldsymbol{\xi}\|_{0, r^{\prime}, \Omega}+\|\boldsymbol{d i v} \boldsymbol{\xi}\|_{0, r^{\prime}, \Omega} \leq C\left\|P_{h}^{0} \boldsymbol{f}-\boldsymbol{f}\right\|_{0, r^{\prime}, \Omega}$.

Thus, from these last relations and (18), we get

$$
\left(\boldsymbol{d i v}\left[\left(\boldsymbol{\sigma}_{h}-p_{h} \boldsymbol{I}\right)+\boldsymbol{\xi}-(\boldsymbol{\sigma}-p \boldsymbol{I})\right], \boldsymbol{v}\right)=0, \forall \boldsymbol{v} \in\left[L^{r}(\Omega)\right]^{2} .
$$

Hence, using the above mentioned inf-sup condition in Lemma 5, we obtain

$$
\begin{aligned}
\beta^{*}\left\|\left(\boldsymbol{\sigma}_{h}-\boldsymbol{\sigma}+\boldsymbol{\xi}, p_{h}-p\right)\right\|_{\Sigma} & \leq \sup _{\boldsymbol{s} \in T} \frac{\left(\boldsymbol{s}, \boldsymbol{\sigma}_{h}-\boldsymbol{\sigma}+\boldsymbol{\xi}\right)}{\|\boldsymbol{s}\|_{T}} \\
& \leq \sup _{\boldsymbol{s} \in T} \frac{\left(\boldsymbol{s}, \boldsymbol{\sigma}_{h}-\boldsymbol{\sigma}\right)}{\|\boldsymbol{s}\|_{T}}+\sup _{\boldsymbol{s} \in T} \frac{(\boldsymbol{s}, \boldsymbol{\xi})}{\|\boldsymbol{s}\|_{T}} .
\end{aligned}
$$


This last inequality together with (13), lead to

$$
\begin{aligned}
&\left\|\left(\boldsymbol{\sigma}_{h}-\boldsymbol{\sigma}+\boldsymbol{\xi}, p_{h}-p\right)\right\|_{\Sigma} \leq C\left(\boldsymbol{R}_{1 *}+\right. \\
&\left.\sup _{\boldsymbol{s} \in T} \frac{\left(\mathcal{A}\left(\boldsymbol{t}_{h}\right)-\mathcal{A}(\boldsymbol{t}), \boldsymbol{s}\right)}{\|\boldsymbol{s}\|_{T}}+\left\|\boldsymbol{f}-P_{h}^{0} \boldsymbol{f}\right\|_{0, r^{\prime}, \Omega}\right) .
\end{aligned}
$$

On the other hand,

$$
\begin{gathered}
\left\|\boldsymbol{\sigma}_{h}-\underset{\sim}{\boldsymbol{\sigma}}\right\|_{\Sigma}=\left\|\left(\boldsymbol{\sigma}_{h}-\boldsymbol{\sigma}, p_{h}-p\right)\right\|_{\Sigma} \leq\left\|\left(\boldsymbol{\sigma}_{h}-\boldsymbol{\sigma}+\boldsymbol{\xi}, p_{h}-p\right)\right\|_{\Sigma} \\
+\|(\boldsymbol{\xi}, 0)\|_{\Sigma}
\end{gathered}
$$

and

$$
\|(\boldsymbol{\xi}, 0)\|_{\Sigma}=\left(\|\boldsymbol{\xi}\|_{0, r^{\prime}, \Omega}^{r^{\prime}}+\|\boldsymbol{d i v} \boldsymbol{\xi}\|_{0, r^{\prime}, \Omega}^{r^{\prime}}\right)^{1 / r^{\prime}} \leq C\left\|P_{h}^{0} \boldsymbol{f}-\boldsymbol{f}\right\|_{0, r^{\prime}, \Omega} .
$$

Then, from (19), we get

$$
\begin{aligned}
\left\|\boldsymbol{\sigma}_{h}-\underset{\sim}{\boldsymbol{\sigma}}\right\|_{\Sigma} \leq C\left(\boldsymbol{R}_{1 *}\right. & +\left\|P_{h}^{0} \boldsymbol{f}-\boldsymbol{f}\right\|_{0, r^{\prime}, \Omega} \\
& \left.+\sup _{\boldsymbol{s} \in \boldsymbol{T}} \frac{\left(\mathcal{A}\left(\boldsymbol{t}_{h}\right)-\mathcal{A}(\boldsymbol{t}), \boldsymbol{s}\right)}{\|\boldsymbol{s}\|_{T}}\right) .
\end{aligned}
$$

Finally, using (16), (17) and (20), we get

$$
\begin{aligned}
\left(\mathcal{A}\left(\boldsymbol{t}_{h}\right)-\mathcal{A}(\boldsymbol{t}), \boldsymbol{t}_{h}-\boldsymbol{t}\right) \leq & \boldsymbol{R}_{1 *}\left\|\boldsymbol{t}_{h}-\boldsymbol{t}\right\|_{T}+\boldsymbol{R}_{2 *}\left\|\boldsymbol{\sigma}_{\sim}-\underset{\sim}{\boldsymbol{\sigma}}\right\|_{\Sigma} \\
& +\boldsymbol{R}_{3 *}\left\|\boldsymbol{u}_{\sim}-\underset{\sim}{\boldsymbol{u}}\right\|_{M} \\
\leq & C\left\{\boldsymbol{R}_{1 *}\left\|\boldsymbol{t}_{h}-\boldsymbol{t}\right\|_{T}+\boldsymbol{R}_{2 *} \boldsymbol{R}_{1 *}\right. \\
& +\boldsymbol{R}_{2 *}\left\|P_{h}^{0} \boldsymbol{f}-\boldsymbol{f}\right\|_{0, r^{\prime}, \Omega} \\
& +\boldsymbol{R}_{2 *} \sup _{\boldsymbol{s} \in \boldsymbol{T}} \frac{\left(\boldsymbol{\mathcal { A }}\left(\boldsymbol{t}_{h}\right)-\mathcal{A}(\boldsymbol{t}), \boldsymbol{s}\right)}{\|\boldsymbol{s}\|_{T}} \\
& \left.+\boldsymbol{R}_{3 *} \boldsymbol{R}_{2 *}+\boldsymbol{R}_{3 *}\left\|\boldsymbol{t}_{h}-\boldsymbol{t}\right\|_{T}\right\} .
\end{aligned}
$$

This ends the proof of the previous proposition.

As it was mentioned above, the upper bounds of the errors depend on the parameter $r>1$. On the other hand, to distinguish the two models, we set: $\delta=0$ for power law and $\delta=1$ for Carreau law.

We first consider the case where the parameter $r$ verify $1<r<2$. 
Theorem 10. Let $(\boldsymbol{t}, \underset{\sim}{\boldsymbol{\sigma}}, \underset{\sim}{\boldsymbol{u}})$ and $\left(\boldsymbol{t}_{h}, \boldsymbol{\sigma}_{h},{\underset{\sim}{\boldsymbol{u}}}_{h}\right)$ be the solution of problems (5) and (7), respectively. Suppose that $1<r<2$, then there exists a constant $C$ independent of $h$ such that

$$
\begin{aligned}
&\left\|\boldsymbol{t}-\boldsymbol{t}_{h}\right\|_{T} \leq C\left(\sum_{i=1}^{3} \boldsymbol{R}_{i *}+\boldsymbol{R}_{2 *}^{r / 2}+\left\|\boldsymbol{f}-P_{h}^{0} \boldsymbol{f}\right\|_{0, r^{\prime}, \Omega}\right), \\
& \| \underset{\sim}{\boldsymbol{\sigma}-\boldsymbol{\sigma}_{h} \|_{\Sigma} \leq} C\left(\boldsymbol{R}_{1 *}+\sum_{i=1}^{3} \boldsymbol{R}_{i *}^{2 / r^{\prime}}+\boldsymbol{R}_{2 *}^{r / r^{\prime}}\right. \\
&\left.+\left\|\boldsymbol{f}-P_{h}^{0} \boldsymbol{f}\right\|_{0, r^{\prime}, \Omega}+\left\|\boldsymbol{f}-P_{h}^{0} \boldsymbol{f}\right\|_{0, r^{\prime}, \Omega}^{2 / r^{\prime}}\right), \\
& \| \underset{\sim}{\boldsymbol{u}-\boldsymbol{u}_{h} \|_{M} \leq C}\left(\sum_{i=1}^{3} \boldsymbol{R}_{i *}+\boldsymbol{R}_{2 *}^{r / 2}+\left\|\boldsymbol{f}-P_{h}^{0} \boldsymbol{f}\right\|_{0, r^{\prime}, \Omega}\right) .
\end{aligned}
$$

Proof. Owing to Lemma 3.1 and Lemma 3.3 in Farhloul-Zine [13], we have

$$
\begin{aligned}
\left(\mathcal{A}\left(\boldsymbol{t}_{h}\right)-\mathcal{A}(\boldsymbol{t}), \boldsymbol{t}_{h}-\boldsymbol{t}\right) \geq C & \left\{\frac{\left\|\boldsymbol{t}_{h}-\boldsymbol{t}\right\|_{0, r, \Omega}^{2}}{\delta+\left\|\boldsymbol{t}_{h}\right\|_{0, r, \Omega}^{2-r}+\|\boldsymbol{t}\|_{0, r, \Omega}^{2-r}}\right. \\
& \left.+\int_{\Omega}\left|\mathcal{A}\left(\boldsymbol{t}_{h}\right)-\mathcal{A}(\boldsymbol{t})\right|\left|\boldsymbol{t}_{h}-\boldsymbol{t}\right| d x\right\},
\end{aligned}
$$

and

$$
\left\|\mathcal{A}\left(\boldsymbol{t}_{h}\right)-\mathcal{A}(\boldsymbol{t})\right\|_{0, r^{\prime}, \Omega} \leq C\left[\int_{\Omega}\left|\mathcal{A}\left(\boldsymbol{t}_{h}\right)-\mathcal{A}(\boldsymbol{t})\right|\left|\boldsymbol{t}_{h}-\boldsymbol{t}\right| d x\right]^{1 / r^{\prime}} .
$$

Then, we get from (12),

$$
\begin{gathered}
\frac{\left\|\boldsymbol{t}_{h}-\boldsymbol{t}\right\|_{T}^{2}}{\delta+\left\|\boldsymbol{t}_{h}\right\|_{T}^{2-r}+\|\boldsymbol{t}\|_{T}^{2-r}}+\int_{\Omega}\left|\mathcal{A}\left(\boldsymbol{t}_{h}\right)-\mathcal{A}(\boldsymbol{t})\right|\left|\boldsymbol{t}_{h}-\boldsymbol{t}\right| d x \\
\leq C\left\{\left(\boldsymbol{R}_{1 *}+\boldsymbol{R}_{3 *}\right)\left\|\boldsymbol{t}_{h}-\boldsymbol{t}\right\|_{T}+\boldsymbol{R}_{2 *}\left\|\boldsymbol{f}-P_{h}^{0} \boldsymbol{f}\right\|_{0, r^{\prime}, \Omega}\right. \\
\left.+\left(\boldsymbol{R}_{1 *}+\boldsymbol{R}_{3 *}\right) \boldsymbol{R}_{2 *}+\boldsymbol{R}_{2 *} \sup _{\boldsymbol{s} \in \boldsymbol{T}} \frac{\left(\mathcal{A}(\boldsymbol{t})-\mathcal{A}\left(\boldsymbol{t}_{h}\right), \boldsymbol{s}\right)}{\|\boldsymbol{s}\|_{T}}\right\} .
\end{gathered}
$$

On the other hand, using stability conditions (see Farhloul-Zine [13]),

$$
\|\boldsymbol{t}\|_{T} \leq C(\boldsymbol{f}) \quad \text { and } \quad\left\|\boldsymbol{t}_{h}\right\|_{T} \leq C(\boldsymbol{f}),
$$

and (24), we get

$$
\left\|\boldsymbol{t}_{h}-\boldsymbol{t}\right\|_{T}^{2}+\int_{\Omega}\left|\mathcal{A}\left(\boldsymbol{t}_{h}\right)-\mathcal{A}(\boldsymbol{t})\right|\left|\boldsymbol{t}_{h}-\boldsymbol{t}\right| d x
$$




$$
\begin{aligned}
& \leq C\left\{\left(\boldsymbol{R}_{1 *}+\boldsymbol{R}_{3 *}\right)\left\|\boldsymbol{t}-\boldsymbol{t}_{h}\right\|_{T}+\boldsymbol{R}_{2 *}\left\|\boldsymbol{f}-P_{h}^{0} \boldsymbol{f}\right\|_{0, r^{\prime}, \Omega}\right. \\
& \left.+\boldsymbol{R}_{2 *}\left(\boldsymbol{R}_{1 *}+\boldsymbol{R}_{3 *}\right)+\boldsymbol{R}_{2 *}\left[\int_{\Omega}\left|\mathcal{A}\left(\boldsymbol{t}_{h}\right)-\mathcal{A}(\boldsymbol{t})\right|\left|\boldsymbol{t}_{h}-\boldsymbol{t}\right| d x\right]^{1 / r^{\prime}}\right\} .
\end{aligned}
$$

Now, using the Young inequality, we obtain, $\forall \varepsilon>0$ and $\forall \bar{\varepsilon}>0$,

$$
\begin{aligned}
& \left\|\boldsymbol{t}_{h}-\boldsymbol{t}\right\|_{T}^{2}+\int_{\Omega}\left|\mathcal{A}\left(\boldsymbol{t}_{h}\right)-\mathcal{A}(\boldsymbol{t})\right|\left|\boldsymbol{t}_{h}-\boldsymbol{t}\right| d x \\
& \leq C\left\{\varepsilon^{-1}\left(\boldsymbol{R}_{1 *}+\boldsymbol{R}_{3 *}\right)^{2}+\varepsilon\left\|\boldsymbol{t}-\boldsymbol{t}_{h}\right\|_{T}^{2}\right. \\
& +\boldsymbol{R}_{2 *}\left\|\boldsymbol{f}-P_{h}^{0} \boldsymbol{f}\right\|_{0, r^{\prime}, \Omega}+\boldsymbol{R}_{2 *}\left(\boldsymbol{R}_{1 *}+\boldsymbol{R}_{3 *}\right) \\
& \left.+(\bar{\varepsilon})^{-r} \boldsymbol{R}_{2 *}^{r}+(\bar{\varepsilon})^{r^{\prime}} \int_{\Omega}\left|A(\boldsymbol{t})-A\left(\boldsymbol{t}_{h}\right)\right|\left|\boldsymbol{t}-\boldsymbol{t}_{h}\right| d x\right\} .
\end{aligned}
$$

Thus, for an adequate choice of $\varepsilon$ and $\bar{\varepsilon}$, we get

$$
\begin{aligned}
\left\|\boldsymbol{t}_{h}-\boldsymbol{t}\right\|_{T}^{2}+ & \int_{\Omega}\left|\mathcal{A}\left(\boldsymbol{t}_{h}\right)-\mathcal{A}(\boldsymbol{t})\right|\left|\boldsymbol{t}_{h}-\boldsymbol{t}\right| d x \leq C\left\{\left(\boldsymbol{R}_{1 *}+\boldsymbol{R}_{3 *}\right)^{2}\right. \\
& \left.+\boldsymbol{R}_{2 *}\left\|\boldsymbol{f}-P_{h}^{0} \boldsymbol{f}\right\|_{0, r^{\prime}, \Omega}+\boldsymbol{R}_{2 *}\left(\boldsymbol{R}_{1 *}+\boldsymbol{R}_{3 *}\right)+\boldsymbol{R}_{2 *}^{r}\right\} .
\end{aligned}
$$

This implies, in particular, the expected result in equation (21), namely:

$$
\left\|\boldsymbol{t}-\boldsymbol{t}_{h}\right\|_{T} \leq C\left(\sum_{i=1}^{3} \boldsymbol{R}_{i *}+\boldsymbol{R}_{2 *}^{r / 2}+\left\|\boldsymbol{f}-P_{h}^{0} \boldsymbol{f}\right\|_{0, r^{\prime}, \Omega}\right),
$$

and

$$
\begin{aligned}
& \int_{\Omega}\left|\mathcal{A}\left(\boldsymbol{t}_{h}\right)-\mathcal{A}(\boldsymbol{t})\right|\left|\boldsymbol{t}-\boldsymbol{t}_{h}\right| d x \\
& \quad \leq C\left(\sum_{i=1}^{3} \boldsymbol{R}_{i *}^{2}+\boldsymbol{R}_{2 *}^{r}+\left\|\boldsymbol{f}-P_{h}^{0} \boldsymbol{f}\right\|_{0, r^{\prime}, \Omega}^{2}\right) .
\end{aligned}
$$

Finally, to obtain the expected estimate (22), it suffices to use the inequalities (20), (24) and (25). And the estimate (23) is a direct consequence of (17) and (21).

After the study of the case $1<r<2$, we will now consider the case $r \geq 2$.

Theorem 11. Let $(\boldsymbol{t}, \underset{\sim}{\boldsymbol{\sigma}} \underset{\sim}{\boldsymbol{u}})$ and $\left(\boldsymbol{t}_{h}, \underset{\sim}{\boldsymbol{\sigma}_{h}}, \boldsymbol{u}_{h}\right)$ be the solution of problems (5) and (7), respectively. Suppose that $r \geq 2$, then there exists a constant $C$ independent of $h$ such that

$$
\begin{gathered}
\left\|\boldsymbol{t}-\boldsymbol{t}_{h}\right\|_{T} \leq C\left(\sum_{i=1}^{3} \boldsymbol{R}_{i *}^{r^{\prime} / r}+\boldsymbol{R}_{2 *}+\boldsymbol{R}_{2 *}^{2 / r}\right. \\
\left.+\left\|\boldsymbol{f}-P_{h}^{0} \boldsymbol{f}\right\|_{0, r^{\prime}, \Omega}\right)
\end{gathered}
$$




$$
\begin{aligned}
&\left\|\underset{\sim}{\boldsymbol{\sigma}}-\boldsymbol{\sigma}_{h}\right\|_{\Sigma} \leq C\left(\boldsymbol{R}_{1 *}^{r^{\prime} / 2}+\boldsymbol{R}_{3 *}^{r^{\prime} / 2}+\sum_{i=1}^{3} \boldsymbol{R}_{i *}\right. \\
&\left.+\left\|\boldsymbol{f}-P_{h}^{0} \boldsymbol{f}\right\|_{0, r^{\prime}, \Omega}\right), \\
& \| \underset{\sim}{\boldsymbol{u}}-{\underset{\sim}{\boldsymbol{u}_{h}} \|_{M} \leq C}\left(\sum_{i=1}^{3} \boldsymbol{R}_{i *}^{r^{\prime} / r}+\boldsymbol{R}_{2 *}+\boldsymbol{R}_{2 *}^{2 / r}\right. \\
&\left.+\left\|\boldsymbol{f}-P_{h}^{0} \boldsymbol{f}\right\|_{0, r^{\prime}, \Omega}\right) .
\end{aligned}
$$

Proof. Using Lemma 3.2 in Farhloul-Zine [13], we get

$$
\begin{aligned}
&\left(\mathcal{A}\left(\boldsymbol{t}_{h}\right)-\mathcal{A}(\boldsymbol{t}), \boldsymbol{t}_{h}-\boldsymbol{t}\right) \geq C\left(\left\|\boldsymbol{t}_{h}-\boldsymbol{t}\right\|_{0, r, \Omega}^{r}\right. \\
&\left.\quad+\int_{\Omega}\left(\delta+\left|\boldsymbol{t}_{h}\right|^{r-2}+|\boldsymbol{t}|^{r-2}\right)\left|\boldsymbol{t}_{h}-\boldsymbol{t}\right|^{2} d x\right)
\end{aligned}
$$

and

$$
\begin{aligned}
\| \mathcal{A}\left(\boldsymbol{t}_{h}\right)- & \mathcal{A}(\boldsymbol{t}) \|_{0, r^{\prime}, \Omega} \\
& \leq C\left[\int_{\Omega}\left(\delta+\left|\boldsymbol{t}_{h}\right|^{r-2}+|\boldsymbol{t}|^{r-2}\right)\left|\boldsymbol{t}_{h}-\boldsymbol{t}\right|^{2} d x\right]^{1 / 2} \\
& \times\left[\delta+\left\|\boldsymbol{t}_{h}\right\|_{0, r, \Omega}^{(r-2) / 2}+\|\boldsymbol{t}\|_{0, r, \Omega}^{(r-2) / 2}\right] .
\end{aligned}
$$

To simplify the notations, we set

$$
\boldsymbol{\Lambda}=\left\|\boldsymbol{t}-\boldsymbol{t}_{h}\right\|_{T}^{r}+\int_{\Omega}\left(\delta+\left|\boldsymbol{t}_{h}\right|^{r-2}+|\boldsymbol{t}|^{r-2}\right)\left|\boldsymbol{t}-\boldsymbol{t}_{h}\right|^{2} d x .
$$

Then, from (12), we have

$$
\begin{aligned}
\boldsymbol{\Lambda} \leq C & \left(\boldsymbol{R}_{1 *}+\boldsymbol{R}_{3 *}\right)\left(\left\|\boldsymbol{t}_{h}-\boldsymbol{t}\right\|_{T}+\boldsymbol{R}_{2 *}\right)+\boldsymbol{R}_{2 *}\left\|\boldsymbol{f}-P_{h}^{0} \boldsymbol{f}\right\|_{0, r^{\prime}, \Omega} \\
& \left.+\boldsymbol{R}_{2 *}\left\|\boldsymbol{A}\left(\boldsymbol{t}_{h}\right)-\mathcal{A}(\boldsymbol{t})\right\|_{0, r^{\prime}, \Omega}\right\}
\end{aligned}
$$

And then, from (29), we get

$$
\begin{aligned}
\boldsymbol{\Lambda} \leq C & \left\{\boldsymbol{R}_{1 *}+\boldsymbol{R}_{3 *}\right)\left(\left\|\boldsymbol{t}_{h}-\boldsymbol{t}\right\|_{T}+\boldsymbol{R}_{2 *}\right)+\boldsymbol{R}_{2 *}\left\|\boldsymbol{f}-P_{h}^{0} \boldsymbol{f}\right\|_{0, r^{\prime}, \Omega} \\
& +\boldsymbol{R}_{2 *}\left[\int_{\Omega}\left(\delta+\left|\boldsymbol{t}_{h}\right|^{r-2}+|\boldsymbol{t}|^{r-2}\right)\left|\boldsymbol{t}_{h}-\boldsymbol{t}\right|^{2} d x\right]^{1 / 2} \\
& \left.\times\left(\delta+\left\|\boldsymbol{t}_{h}\right\|_{T}^{(r-2) / 2}+\|\boldsymbol{t}\|_{T}^{(r-2) / 2}\right)\right\} .
\end{aligned}
$$


And again, using Young's inequality with two parameters $\varepsilon$ and $\bar{\varepsilon}$, we get

$$
\begin{aligned}
\boldsymbol{\Lambda} & \leq C\left\{\varepsilon^{r}\left\|\boldsymbol{t}_{h}-\boldsymbol{t}\right\|_{T}^{r}+\varepsilon^{-r^{\prime}}\left(\boldsymbol{R}_{1 *}+\boldsymbol{R}_{3 *}\right)^{r^{\prime}}+\boldsymbol{R}_{2 *}\left\|\boldsymbol{f}-P_{h}^{0} \boldsymbol{f}\right\|_{0, r^{\prime}, \Omega}\right. \\
& +\left(\boldsymbol{R}_{1 *}+\boldsymbol{R}_{3 *}\right) \boldsymbol{R}_{2 *}+\bar{\varepsilon} \int_{\Omega}\left(\delta+\left|\boldsymbol{t}_{h}\right|^{r-2}+|\boldsymbol{t}|^{r-2}\right)\left|\boldsymbol{t}_{h}-\boldsymbol{t}\right|^{2} d x \\
& \left.+(\bar{\varepsilon})^{-1} \boldsymbol{R}_{2 *}^{2}\left(\delta+\left\|\boldsymbol{t}_{h}\right\|_{T}^{(r-2) / 2}+\|\boldsymbol{t}\|_{T}^{(r-2) / 2}\right)^{2}\right\} .
\end{aligned}
$$

Thus, using the stability conditions $\|\boldsymbol{t}\|_{T} \leq C(\boldsymbol{f})$ and $\left\|\boldsymbol{t}_{h}\right\|_{T} \leq C(\boldsymbol{f})$, we obtain

$$
\begin{aligned}
\boldsymbol{\Lambda} \leq C & \left\{\left(\boldsymbol{R}_{1 *}+\boldsymbol{R}_{3 *}\right)^{r^{\prime}}+\boldsymbol{R}_{2 *}\left\|\boldsymbol{f}-P_{h}^{0} \boldsymbol{f}\right\|_{0, r^{\prime}, \Omega}\right. \\
& \left.+\left(\boldsymbol{R}_{1 *}+\boldsymbol{R}_{3 *}\right) \boldsymbol{R}_{2 *}+\boldsymbol{R}_{2 *}^{2}\right\} .
\end{aligned}
$$

This last inequality leads in particular to

$$
\left\|\boldsymbol{t}-\boldsymbol{t}_{h}\right\|_{T}^{r} \leq C\left\{\left(\boldsymbol{R}_{1 *}+\boldsymbol{R}_{3 *}\right)^{r^{\prime}}+\boldsymbol{R}_{2 *}^{r^{\prime}}+\left\|\boldsymbol{f}-P_{h}^{0} \boldsymbol{f}\right\|_{0, r^{\prime}, \Omega}^{r}+\boldsymbol{R}_{2 *}^{r}+\boldsymbol{R}_{2 *}^{2}\right\} .
$$

And then,

$$
\left\|\boldsymbol{t}-\boldsymbol{t}_{h}\right\|_{T} \leq C\left\{\sum_{i=1}^{3} \boldsymbol{R}_{i *}^{r^{\prime} / r}+\boldsymbol{R}_{2 *}+\boldsymbol{R}_{2 *}^{2 / r}+\left\|\boldsymbol{f}-P_{h}^{0} \boldsymbol{f}\right\|_{0, r^{\prime}, \Omega}\right\},
$$

which is precisely the expected estimate (26).

On the other hand, from (30), we deduce the following estimate

$$
\begin{aligned}
\int_{\Omega}(\delta & \left.+\left|\boldsymbol{t}_{h}\right|^{r-2}+|\boldsymbol{t}|^{r-2}\right)\left|\boldsymbol{t}-\boldsymbol{t}_{h}\right|^{2} d x \leq C\left\{\left(\boldsymbol{R}_{1 *}+\boldsymbol{R}_{3 *}\right)^{r^{\prime}}\right. \\
& \left.+\left(\boldsymbol{R}_{1 *}+\boldsymbol{R}_{3 *}\right)^{2}+\boldsymbol{R}_{2 *}^{2}+\left\|\boldsymbol{f}-P_{h}^{0} \boldsymbol{f}\right\|_{0, r^{\prime}, \Omega}^{2}\right\}
\end{aligned}
$$

and then, using (20), (29) and the fact that $\|\boldsymbol{t}\|_{T}$ and $\left\|\boldsymbol{t}_{h}\right\|_{T}$ are bounded, we get

$$
\left\|\underset{\sim}{\boldsymbol{\sigma}}-{\underset{\sim}{\sim}}_{h}\right\|_{\Sigma} \leq C\left\{\sum_{i=1}^{3} \boldsymbol{R}_{i *}+\boldsymbol{R}_{1 *}^{r^{\prime} / 2}+\boldsymbol{R}_{3 *}^{r^{\prime} / 2}+\left\|\boldsymbol{f}-P_{h}^{0} \boldsymbol{f}\right\|_{0, r^{\prime}, \Omega}\right\},
$$

which is the expected estimate (27).

Finally, the estimation (28) is a consequence of the estimates (17) and (26). 
Finally, the previous results show that to have the a posteriori error estimates of our problem, it suffices to estimate $\boldsymbol{R}_{i *}, i=1,2,3$. To this end, we first precise some notations: for a tensor field $\boldsymbol{\tau} \in \mathbb{R}^{2 \times 2}$ and for a vector field $\boldsymbol{v}=\left(v_{1}, v_{2}\right) \in \mathbb{R}^{2}$,

- $\operatorname{tr}(\boldsymbol{\tau})=\tau_{11}+\tau_{22}, a s(\boldsymbol{\tau})=\tau_{21}-\tau_{12}$,

- $\operatorname{rot}(\boldsymbol{\tau})=\left(\frac{\partial \tau_{12}}{\partial x_{1}}-\frac{\partial \tau_{11}}{\partial x_{2}}, \frac{\partial \tau_{22}}{\partial x_{1}}-\frac{\partial \tau_{21}}{\partial x_{2}}\right)$,

- $\operatorname{Curl}(\boldsymbol{v})=\left(\begin{array}{ll}\frac{\partial v_{1}}{\partial x_{2}} & -\frac{\partial v_{1}}{\partial x_{1}} \\ \frac{\partial v_{2}}{\partial x_{2}} & -\frac{\partial v_{2}}{\partial x_{1}}\end{array}\right)$,

- and $[[g]]_{E}$ stands for the jump of function $g$ across an edge E.

We also recall the following Helmholtz decomposition of a tensor field in $\boldsymbol{\Sigma}$.

Proposition 12. Let $\underset{\sim}{\boldsymbol{\tau}} \in \boldsymbol{\Sigma}$. Then there exist $\boldsymbol{z} \in\left[W^{2, r^{\prime}}(\Omega)\right]^{2}$ and $\boldsymbol{\psi} \in\left[W^{1, r^{\prime}}(\Omega)\right]^{2}$ such that

$$
\boldsymbol{\tau}-q \boldsymbol{I}=\nabla \boldsymbol{z}+\operatorname{Curl} \boldsymbol{\psi},
$$

with the estimate

$$
\|\boldsymbol{z}\|_{2, r^{\prime}, \Omega}+\|\boldsymbol{\psi}\|_{1, r^{\prime}, \Omega} \leq C\|\boldsymbol{\tau}\|_{\Sigma}
$$

Proof. To prove this result it is sufficient to apply Theorem 1.1 of Creusé et al. [8] to each row of the tensor $\boldsymbol{\tau}-q \boldsymbol{I}$, i.e. the two vector fields $\left(\tau_{11}-q, \tau_{12}\right)$ and $\left(\tau_{21}, \tau_{22}-q\right)$.

Lemma 13. For every $\underset{\sim}{\tau} \in \boldsymbol{\Sigma}$, we have

$$
\begin{aligned}
\left\langle\boldsymbol{R}_{2}, \underset{\sim}{\boldsymbol{\tau}}>\right. & =\sum_{K \in \mathcal{T}_{h}}\left(\boldsymbol{t}_{h}+\boldsymbol{\omega}_{h}, \nabla \boldsymbol{z}-\Pi_{h}(\nabla \boldsymbol{z})\right) \\
& \left.+\sum_{K \in \mathcal{T}_{h}}\left(\operatorname{tr}\left(\boldsymbol{t}_{h}\right)\right), q\right) \\
& +\sum_{K \in \mathcal{T}_{h}}\left(\operatorname{rot}\left(\boldsymbol{t}_{h}+\boldsymbol{\omega}_{h}\right), \boldsymbol{\psi}-I_{c l}(\boldsymbol{\psi})\right) \\
& \left.-\sum_{E \in E_{h}}<\left[\left(\boldsymbol{t}_{h}+\boldsymbol{\omega}_{h}\right) \underline{\boldsymbol{t}}\right]\right]_{E}, \boldsymbol{\psi}-I_{c l}(\boldsymbol{\psi})>_{E},
\end{aligned}
$$

where

- $(\boldsymbol{z}, \boldsymbol{\psi}) \in\left[W^{2, r^{\prime}}(\Omega)\right]^{2} \times\left[W^{1, r^{\prime}}(\Omega)\right]^{2}$ denotes the Helmholtz decomposition of $\underset{\sim}{\boldsymbol{\tau}} \in \boldsymbol{\Sigma}$, 
- $I_{c l}(\boldsymbol{\psi})$ is the Clément interpolant of $\boldsymbol{\psi}$ (see Clément [7]),

- $E_{h}$ denotes the set of all edges of the triangulation $\mathcal{T}_{h}$,

- $\left[\left[\left(\boldsymbol{t}_{h}+\boldsymbol{\omega}_{h}\right) \underline{\boldsymbol{t}}\right]\right]_{E}$ denotes the tangential jump of $\boldsymbol{t}_{h}+\boldsymbol{\omega}_{h}$ across the edge $E$,

- $\Pi_{h}(\nabla \boldsymbol{z})$ is the Brezzi-Douglas-Marini interpolant of the lowest degree of $\nabla \boldsymbol{z}$ (see Brezzi et al. [5]).

Proof. By (10), we get for every $\underset{\sim}{\boldsymbol{\tau}} \in \boldsymbol{\Sigma}$,

$$
<\boldsymbol{R}_{2}, \underset{\sim}{\boldsymbol{\tau}}>=\left(\boldsymbol{t}_{h}+\boldsymbol{\omega}_{h}, \boldsymbol{\tau}\right)+\left(\operatorname{div}(\boldsymbol{\tau}-q \boldsymbol{I}), \boldsymbol{u}_{h}\right) .
$$

Then, using the Helmholtz decomposition (31), we get

$$
\begin{aligned}
<\boldsymbol{R}_{2}, \underset{\sim}{\boldsymbol{\tau}}> & =\left(\boldsymbol{t}_{h}+\boldsymbol{\omega}_{h}, \nabla \boldsymbol{z}\right)+\left(\boldsymbol{t}_{h}+\boldsymbol{\omega}_{h}, q \boldsymbol{I}\right) \\
& +\left(\boldsymbol{t}_{h}+\boldsymbol{\omega}_{h}, \operatorname{Curl} \boldsymbol{\psi}\right)+\left(\operatorname{div}(\nabla \boldsymbol{z}), \boldsymbol{u}_{h}\right) .
\end{aligned}
$$

On the other hand, using the properties of $\Pi_{h}(\nabla \boldsymbol{z})$, the Brezzi-Douglas-Marini interpolant, we get

$$
\left(\boldsymbol{d i v}\left(\Pi_{h}(\nabla \boldsymbol{z})\right), \boldsymbol{v}_{h}\right)=\left(\boldsymbol{d i v}(\nabla \boldsymbol{z}), \boldsymbol{v}_{h}\right), \forall \boldsymbol{v}_{h} \in\left[\prod_{K \in \mathcal{T}_{h}} P_{0}(K)\right]^{2} .
$$

Thus, using this last relation and the fact that $\operatorname{tr}\left(\boldsymbol{\omega}_{h}\right)=0$, the equation (34) may be rewritten as follows:

$$
\begin{aligned}
<\boldsymbol{R}_{2}, \boldsymbol{\tau}> & =\left(\boldsymbol{t}_{h}+\boldsymbol{\omega}_{h}, \nabla \boldsymbol{z}\right)+\left(\operatorname{tr}\left(\boldsymbol{t}_{h}\right), q\right) \\
& +\left(\boldsymbol{t}_{h}+\boldsymbol{\omega}_{h}, \operatorname{Curl} \boldsymbol{\psi}\right)+\left(\boldsymbol{d i v}\left(\Pi_{h}(\nabla \boldsymbol{z})\right), \boldsymbol{u}_{h}\right) .
\end{aligned}
$$

Taking successively

$$
\underset{\sim}{\boldsymbol{\tau}_{h}}=\left(\Pi_{h}(\nabla \boldsymbol{z}), 0\right) \in \boldsymbol{\Sigma}_{h} \text { and } \boldsymbol{\tau}_{\sim}=\left(\operatorname{Curl}\left(I_{c l}(\boldsymbol{\psi})\right), 0\right) \in \boldsymbol{\Sigma}_{h}
$$

in the second equation of the discrete problem (7), we obtain

$$
\left(\boldsymbol{t}_{h}+\boldsymbol{\omega}_{h}, \Pi_{h}(\nabla \boldsymbol{z})\right)+\left(\boldsymbol{d i v}\left(\Pi_{h}(\nabla \boldsymbol{z})\right), \boldsymbol{u}_{h}\right)=0
$$

and

$$
\left(\boldsymbol{t}_{h}+\boldsymbol{\omega}_{h}, \operatorname{Curl}\left(I_{c l}(\boldsymbol{\psi})\right)\right)=0
$$


Injecting these two last relations in the right-hand side of (35), we get

$$
\begin{aligned}
\left\langle\boldsymbol{R}_{2}, \boldsymbol{\tau}>\right. & =\left(\boldsymbol{t}_{h}+\boldsymbol{\omega}_{h}, \nabla \boldsymbol{z}-\Pi_{h}(\nabla \boldsymbol{z})\right)+\left(\operatorname{tr}\left(\boldsymbol{t}_{h}\right), q\right) \\
& +\left(\boldsymbol{t}_{h}+\boldsymbol{\omega}_{h}, \operatorname{Curl}\left(\boldsymbol{\psi}-I_{c l}(\boldsymbol{\psi})\right)\right) .
\end{aligned}
$$

Thus, using Green's formula, we obtain

$$
\begin{aligned}
<\boldsymbol{R}_{2}, \underset{\sim}{\boldsymbol{\tau}}>= & \left(\boldsymbol{t}_{h}+\boldsymbol{\omega}_{h}, \nabla \boldsymbol{z}-\Pi_{h}(\nabla \boldsymbol{z})\right)+\left(\operatorname{tr}\left(\boldsymbol{t}_{h}\right), q\right) \\
& +\sum_{K \in \mathcal{T}_{h}}\left[\left(\operatorname{rot}\left(\boldsymbol{t}_{h}+\boldsymbol{\omega}_{h}\right), \boldsymbol{\psi}-I_{c l}(\boldsymbol{\psi})\right)\right] \\
& -\sum_{K \in \mathcal{T}_{h}}\left[\left\langle\left(\boldsymbol{t}_{h}+\boldsymbol{\omega}_{h}\right) \underline{\boldsymbol{t}}, \boldsymbol{\psi}-I_{c l}(\boldsymbol{\psi})\right\rangle_{\partial K}\right] \\
= & \sum_{K \in \mathcal{T}_{h}}\left[\left(\boldsymbol{t}_{h}+\boldsymbol{\omega}_{h}, \nabla \boldsymbol{z}-\Pi_{h}(\nabla \boldsymbol{z})\right)+\left(\operatorname{tr}\left(\boldsymbol{t}_{h}\right), q\right)\right] \\
& +\sum_{K \in \mathcal{T}_{h}}\left[\left(\operatorname{rot}\left(\boldsymbol{t}_{h}+\boldsymbol{\omega}_{h}\right), \boldsymbol{\psi}-I_{c l}(\boldsymbol{\psi})\right)\right] \\
& \left.-\sum_{E \in E_{h}}<\llbracket\left[\left(\boldsymbol{t}_{h}+\boldsymbol{\omega}_{h}\right) \underline{\boldsymbol{t}}\right]\right]_{E}, \boldsymbol{\psi}-I_{c l}(\boldsymbol{\psi})>_{E} .
\end{aligned}
$$

Remark 14. The summation

$$
\sum_{E \in E_{h}}<\left[\left[\left(\boldsymbol{t}_{h}+\boldsymbol{\omega}_{h}\right) \underline{\boldsymbol{t}}\right]\right]_{E}, \boldsymbol{\psi}-I_{c l}(\boldsymbol{\psi})>_{E}
$$

appearing in the right-hand side of formula (33) contains the terms

$$
<\left[\left[\left(\boldsymbol{t}_{h}+\boldsymbol{\omega}_{h}\right) \underline{\boldsymbol{t}}\right]\right]_{E}, \boldsymbol{\psi}-I_{c l}(\boldsymbol{\psi})>_{E},
$$

where $E$ is an edge of the triangulation $\mathcal{T}_{h}$ contained in the boundary of $\Omega$; in this case it must be understood that $\left(\boldsymbol{t}_{h}+\boldsymbol{\omega}_{h}\right) \underline{\boldsymbol{t}}$ is 0 outside of $\Omega$.

We are now able to give upper bounds of $\boldsymbol{R}_{1 *}, \boldsymbol{R}_{2 *}$ and $\boldsymbol{R}_{3 *}$. These upper bounds will be functions of the error indicators $\eta_{1}, \eta_{2}$ and $\eta_{3}$. More precisely, we have the following results.

Theorem 15. There exists a constant $C$ independent of $h$ such that

$$
\boldsymbol{R}_{1 *} \leq\left(\sum_{K \in \mathcal{T}_{h}} \eta_{1}(K)^{r^{\prime}}\right)^{1 / r^{\prime}},
$$




$$
\begin{aligned}
& \boldsymbol{R}_{2 *} \leq C\left(\sum_{K \in \mathcal{T}_{h}} \eta_{2}(K)^{r}\right)^{1 / r}, \\
& \boldsymbol{R}_{3 *} \leq C\left(\sum_{K \in \mathcal{T}_{h}} \eta_{3}(K)^{r^{\prime}}\right)^{1 / r^{\prime}},
\end{aligned}
$$

where $\eta_{1}(K), \eta_{2}(K)$ and $\eta_{3}(K)$ are the local estimators given by

$$
\begin{aligned}
\eta_{1}(K)^{r^{\prime}} & =\left\|\mathcal{A}\left(\boldsymbol{t}_{h}\right)-\boldsymbol{\sigma}_{h}\right\|_{0, r^{\prime}, K}^{r^{\prime}}, \\
\eta_{2}(K)^{r} & =h_{K}^{r}\left[\left\|\boldsymbol{t}_{h}+\boldsymbol{\omega}_{h}\right\|_{0, r, K}^{r}+\left\|\operatorname{rot}\left(\boldsymbol{t}_{h}+\boldsymbol{\omega}_{h}\right)\right\|_{0, r, K}^{r}\right] \\
& \left.+\left\|\operatorname{tr}\left(\boldsymbol{t}_{h}\right)\right\|_{0, r, K}^{r}+\sum_{E \in \partial K} h_{E} \|\left[\left[\boldsymbol{t}_{h}+\boldsymbol{\omega}_{h}\right) \underline{\boldsymbol{t}}\right]\right]_{E} \|_{0, r, E}^{r}, \\
\eta_{3}(K)^{r^{\prime}} & =\left\|\boldsymbol{f}-P_{h}^{0} \boldsymbol{f}\right\|_{0, r^{\prime}, K}^{r^{\prime}}+\left\|a s\left(\boldsymbol{\sigma}_{h}\right)\right\|_{0, r^{\prime}, K}^{r^{\prime}} .
\end{aligned}
$$

Proof. It follows from (9) that for every $\boldsymbol{s} \in \boldsymbol{T}$,

$$
\left|<\boldsymbol{R}_{1}, \boldsymbol{s}>\right| \leq\left(\sum_{K \in \mathcal{T}_{h}}\left\|\mathcal{A}\left(\boldsymbol{t}_{h}\right)-\boldsymbol{\sigma}_{h}\right\|_{0, r^{\prime}, K}^{r^{\prime}}\right)^{1 / r^{\prime}}\|\boldsymbol{s}\|_{0, r, \Omega}
$$

and then,

$$
\sup _{\boldsymbol{s} \in \boldsymbol{T}} \frac{\left|<\boldsymbol{R}_{1}, \boldsymbol{s}>\right|}{\|\boldsymbol{s}\|_{T}} \leq\left(\sum_{K \in \mathcal{T}_{h}}\left\|\mathcal{A}\left(\boldsymbol{t}_{h}\right)-\boldsymbol{\sigma}_{h}\right\|_{0, r^{\prime}, K}^{r^{\prime}}\right)^{1 / r^{\prime}} .
$$

Which is precisely the estimate (36). To show the estimate (37), we will use (33) obtained in Lemma 13. This inequality leads, for every $\underset{\sim}{\boldsymbol{\tau}} \in \boldsymbol{\Sigma}$, to

$$
\begin{aligned}
& \left|<\boldsymbol{R}_{2}, \underset{\sim}{\boldsymbol{\tau}}>\right| \leq \sum_{K \in \mathcal{T}_{h}}\left\|\boldsymbol{t}_{h}+\boldsymbol{\omega}_{h}\right\|_{0, r, K}\left\|\nabla \boldsymbol{z}-\Pi_{h}(\nabla \boldsymbol{z})\right\|_{0, r^{\prime}, K} \\
+ & \sum_{K \in \mathcal{T}_{h}}\left\|\operatorname{tr}\left(\boldsymbol{t}_{h}\right)\right\|_{0, r, K}\|q\|_{0, r^{\prime}, K} \\
+ & \sum_{K \in \mathcal{T}_{h}}^{\| r o t}\left(\boldsymbol{t}_{h}+\boldsymbol{\omega}_{h}\right)\left\|_{0, r, K}\right\| \boldsymbol{\psi}-I_{c l}(\boldsymbol{\psi}) \|_{0, r^{\prime}, K} \\
+ & \sum_{E \in E_{h}}\left\|\left[\left(\boldsymbol{t}_{h}+\boldsymbol{\omega}_{h}\right) \underline{\boldsymbol{t}}\right]_{E}\right\|_{0, r, E}\left\|\boldsymbol{\psi}-I_{c l}(\boldsymbol{\psi})\right\|_{0, r^{\prime}, E} .
\end{aligned}
$$

Now, by Lemma 3.1 in Verfürth [20], we have

$$
\left\|\boldsymbol{\psi}-I_{c l}(\boldsymbol{\psi})\right\|_{0, r^{\prime}, K} \leq C h_{K}|\boldsymbol{\psi}|_{1, r^{\prime}, \omega_{K}}
$$

and

$$
\left\|\boldsymbol{\psi}-I_{c l}(\boldsymbol{\psi})\right\|_{0, r^{\prime}, E} \leq C h_{E}^{1 / r}|\boldsymbol{\psi}|_{1, r^{\prime}, \omega_{E}}
$$


where $\omega_{K}$ denotes the union of $K$ with all the triangles from the triangulation $T_{h}$ adjacent to the triangle $K, \omega_{E}$ denotes the union of at most two triangles of $T_{h}$ admitting $E$ as a common edge and $|\cdot|_{1, r^{\prime}, \omega}$, the semi-norm of $W^{1, r^{\prime}}(\omega)$.

Thus, using these two last estimates and the fact that

$$
\left\|\nabla \boldsymbol{z}-\Pi_{h}(\nabla \boldsymbol{z})\right\|_{0, r^{\prime}, K} \leq C h_{K}|\nabla \boldsymbol{z}|_{1, r^{\prime}, K},
$$

the above inequality (39) yields

$$
\begin{aligned}
\left|<\boldsymbol{R}_{2}, \underset{\sim}{\boldsymbol{\tau}}>\right| \leq C \sum_{K \in \mathcal{T}_{h}} h_{K}\left\|\boldsymbol{t}_{h}+\boldsymbol{\omega}_{h}\right\|_{0, r, K}|\nabla \boldsymbol{z}|_{1, r^{\prime}, K} \\
+C \sum_{K \in \mathcal{T}_{h}}\left\|\operatorname{tr}\left(\boldsymbol{t}_{h}\right)\right\|_{0, r, K}\|q\|_{0, r^{\prime}, K} \\
+C \sum_{K \in \mathcal{T}_{h}} h_{K}\left\|\operatorname{rot}\left(\boldsymbol{t}_{h}+\boldsymbol{\omega}_{h}\right)\right\|_{0, r, K}|\boldsymbol{\psi}|_{1, r^{\prime}, \omega_{K}} \\
+C \sum_{E \in E_{h}} h_{E}^{1 / r}\left\|\left[\left(\boldsymbol{t}_{h}+\boldsymbol{\omega}_{h}\right) \underline{\boldsymbol{t}}\right]_{E}\right\|_{0, r, E}|\boldsymbol{\psi}|_{1, r^{\prime}, \omega_{E}} \\
\leq C\left(\sum_{K \in \mathcal{T}_{h}} h_{K}^{r}\left\|\boldsymbol{t}_{h}+\boldsymbol{\omega}_{h}\right\|_{0, r, K}^{r}\right)^{1 / r}|\nabla \boldsymbol{z}|_{1, r^{\prime}, \Omega} \\
+C\left(\sum_{K \in \mathcal{T}_{h}}\left\|\operatorname{tr}\left(\boldsymbol{t}_{h}\right)\right\|_{0, r, K}^{r}\right)^{1 / r}\|q\|_{0, r^{\prime}, \Omega} \\
+C\left(\sum_{K \in \mathcal{T}_{h}} h_{K}^{r}\left\|r o t\left(\boldsymbol{t}_{h}+\boldsymbol{\omega}_{h}\right)\right\|_{0, r, K}^{r}\right)^{1 / r}|\boldsymbol{\psi}|_{1, r^{\prime}, \Omega} \\
\left.+C\left(\sum_{E \in E_{h}} h_{E} \|\left[\left(\boldsymbol{t}_{h}+\boldsymbol{\omega}_{h}\right) \underline{\boldsymbol{t}}\right]\right]_{E} \|_{0, r, E}^{r}\right)^{1 / r}|\boldsymbol{\psi}|_{1, r^{\prime}, \Omega}
\end{aligned}
$$

and so

$$
\begin{aligned}
& \left|<\boldsymbol{R}_{2}, \underset{\sim}{\boldsymbol{\tau}}>\right| \leq C\left\{\sum _ { K \in \mathcal { T } _ { h } } \left(h_{K}^{r}\left\|\boldsymbol{t}_{h}+\boldsymbol{\omega}_{h}\right\|_{0, r, K}^{r}+\left\|\operatorname{tr}\left(\boldsymbol{t}_{h}\right)\right\|_{0, r, K}^{r}\right.\right. \\
& \left.\left.+\quad h_{K}^{r}\left\|\operatorname{rot}\left(\boldsymbol{t}_{h}+\boldsymbol{\omega}_{h}\right)\right\|_{0, r, K}^{r}+\sum_{E \subset \partial K} h_{E}\left\|\left[\left[\left(\boldsymbol{t}_{h}+\boldsymbol{\omega}_{h}\right) \underline{\boldsymbol{t}}\right]\right]_{E}\right\|_{0, r, E}^{r}\right)\right\}^{1 / r} \\
& \times \quad\left\{|\nabla \boldsymbol{z}|_{1, r^{\prime}, \Omega}^{r^{\prime}}+\|q\|_{0, r^{\prime}, \Omega}^{r^{\prime}}+|\boldsymbol{\psi}|_{1, r^{\prime}, \Omega}^{r^{\prime}}\right\}^{1 / r^{\prime}} .
\end{aligned}
$$

Therefore, using (32), we obtain

$$
\left|<\boldsymbol{R}_{2}, \underset{\sim}{\boldsymbol{\tau}}>\right| \leq C\left(\sum_{K \in \mathcal{T}_{h}} \eta_{2}(K)^{r}\right)^{1 / r}\|\underset{\sim}{\boldsymbol{\tau}}\|_{\Sigma}
$$


and (37) follows immediately.

It remains to prove (38). By (11), we have for every $\underset{\sim}{\boldsymbol{v}} \in \boldsymbol{M}$,

$$
\begin{aligned}
&\left|<\boldsymbol{R}_{3}, \underset{\sim}{\boldsymbol{v}}>\right| \leq\left(\sum_{K \in \mathcal{T}_{h}}\left\|\boldsymbol{d i v}\left(\boldsymbol{\sigma}_{h}-p_{h} \boldsymbol{I}\right)+\boldsymbol{f}\right\|_{0, r^{\prime}, K}^{r^{\prime}}\right)^{1 / r^{\prime}}\|\boldsymbol{v}\|_{0, r, \Omega} \\
&+C\left(\sum_{K \in \mathcal{T}_{h}}\left\|a s\left(\boldsymbol{\sigma}_{h}\right)\right\|_{0, r^{\prime}, K}^{r^{\prime}}\right)^{1 / r^{\prime}}\|\boldsymbol{\eta}\|_{0, r, \Omega} \\
& \leq C\left(\sum_{K \in \mathcal{T}_{h}}\left\|\boldsymbol{d i v}\left(\boldsymbol{\sigma}_{h}-p_{h} \boldsymbol{I}\right)+\boldsymbol{f}\right\|_{0, r^{\prime}, K}^{r^{\prime}}\right. \\
&\left.+\left\|a s\left(\boldsymbol{\sigma}_{h}\right)\right\|_{0, r^{\prime}, K}^{r^{\prime}}\right)^{1 / r^{\prime}}\left(\|\boldsymbol{v}\|_{0, r, \Omega}^{r}+\|\boldsymbol{\eta}\|_{0, r, \Omega}^{r}\right)^{1 / r} .
\end{aligned}
$$

On the other hand, following the second equation of the discrete problem (7), we have

$$
\boldsymbol{d i v}\left(\boldsymbol{\sigma}_{h}-p_{h} \boldsymbol{I}\right)=-P_{h}^{0} \boldsymbol{f} .
$$

Therefore, for every $\underset{\sim}{\boldsymbol{v}} \in \boldsymbol{M}$,

$$
\left|<\boldsymbol{R}_{3}, \underset{\sim}{\boldsymbol{v}}>\right| \leq C\left(\sum_{K \in \mathcal{T}_{h}}\left\|\boldsymbol{f}-P_{h}^{0} \boldsymbol{f}\right\|_{0, r^{\prime}, K}^{r^{\prime}}+\left\|a s\left(\boldsymbol{\sigma}_{h}\right)\right\|_{0, r^{\prime}, K}^{r^{\prime}}\right)^{1 / r^{\prime}}\|\underset{\sim}{\boldsymbol{v}}\|_{M}
$$

which implies $\boldsymbol{R}_{3 *} \leq C \eta_{3}$, and the proof is completed.

\section{Conclusion}

In this work, we have developed and analyzed a new a posteriori error estimator for a dual mixed finite element approximation of non-Newtonian fluid flow problems. Our mixed method allows to treat, in a unified approach, both the power law and the Carreau law. The estimator justifies an adaptive finite element scheme which refines a given grid only in regions where the error is relatively large. Furthermore, this estimator generalizes the one that we have obtained in the particular case of power law (see, Farhloul and Zine [12]).

\section{References}

[1] J. Baranger and K. Najib, Analyse numérique des écoulements quasinewtoniens dont la viscosité obéit à la loi puissance ou la loi de Carreau, Numer. Math., 58 (1990), 35-49. 
[2] J.W. Barrett and W.B. Liu, Finite element error analysis of a quasiNewtonian flow obeying the Carreau or power law, Numer. Math., 64 (1993), 433-453.

[3] J.W. Barrett and W.B. Liu, Quasi-norm error bounds for the finite element approximation of a non-Newtonian flow, Numer. Math., 68 (1994), 437456.

[4] R.B. Bird, Robert C. Armstrong and Ole Hassager, Eds., Dynamics of Polymeric Liquids. Volume I : Fluid Mechanics, John Wiley \& Sons, Inc., New York, 2nd ed., 1987.

[5] F. Brezzi, J. Douglas, and L.D. Marini, Two families of mixed finite elements for second order elliptic problems, Numer. Math., 47 (1985), 217235.

[6] P.G. Ciarlet, The Finite Element Methods for Elliptic Problems, North Holland, 1978.

[7] P. Clément, Approximation by finite element functions using local regularization, RAIRO Anal. Numer., 2 (1975), 77-84.

[8] E. Creusé, M. Farhloul, and L. Paquet, A posteriori error estimation for the dual mixed finite element method for the p-Laplacian in a polygonal domain, Comput. Methods Appl. Mech. Engrg., 196 (2007), 2570-2582.

[9] V.J. Ervin, J.S. Howell, I. Stanculescu, A dual-mixed approximation method for a three-field model of a nonlinear generalized Stokes problem, Comput. Methods Appl. Mech. Engrg., 197 (2008), 2886-2900.

[10] M. Farhloul, A.M. Zine, A mixed finite element method for a Ladyzhenskaya model, Comput. Methods Appl. Mech. Engrg., 191 (2002), 44974510.

[11] M. Farhloul and A.M. Zine, A mixed finite element method for a quasiNewtonian fluid flow, Numer. Methods Partial Differential Eq., 20 (2004), 803-819.

[12] M. Farhloul, A.M. Zine, A posteriori error estimation for a dual mixed finite element approximation of non-Newtonian fluid flow problems, Int. J. Numer. Anal. Model., 5 (2008), 320-330. 
[13] M. Farhloul and A.M. Zine, A dual-mixed finite element method for quasiNewtonian flows whose viscosity obeys a power law or the Carreau law, Math. Comput. Simulation 141 (2017), 83-95.

[14] G.P. Galdi, An Introduction to the Mathematical Theory of the NavierStokes Equations, Vol. I, Springer-Verlag, Berlin, 1994.

[15] G.N. Gatica, M. Gonzalez, S. Meddahi, A low-order mixed finite element method for a class of quasi-Newtonian Stokes flows. Part I: a priori error analysis, Comput. Methods Appl. Mech. Engrg., 193 (2004), 881-892.

[16] G.N. Gatica, M. Gonzalez, S. Meddahi, A low-order mixed finite element method for a class of quasi-Newtonian Stokes flows. Part II: a posteriori error analysis, Comput. Methods Appl. Mech. Engrg., 193 (2004), 893-911.

[17] O.A. Ladyzhenskaya, New equations for the description of the viscous incompressible fluids and solvability in the large of the boundary value problems for them, Boundary Value Problems of Mathematical Physics V, Providence, RI: American Mathematical Society, 1970.

[18] H. Manouzi and M. Farhloul, Mixed finite element analysis of a non-linear three-fields Stokes model, IMA J. Numer. Anal., 21 (2001), 143-164.

[19] D. Sandri, Sur l'approximation numérique des écoulements quasiNewtoniens dont la viscosité suit la loi puissance ou la loi de Carreau, $M^{2} A N, 27$ (1993), 131-155.

[20] R. Verfürth, A Review of a Posteriori Error Estimation and Adaptive MeshRefinement Techniques, Wiley and Teubner, 1996. 
